\title{
Motion Study of a Swimmer's Hand and Forearm in Freestyle Swimming
}

\author{
Shubham S. Mahajan ${ }^{\dagger}$ and S. P. Wadkar ${ }^{\dagger}$ \\ †Department of Mechanical Engineering, MIT College of Engineering, Kothrud, Pune, Maharashtra 411038, India
}

Accepted 02 March 2016, Available online 15 March 2016, Special Issue-4 (March 2016)

\begin{abstract}
Swimming being one of the very well known athletic sports, a lot of efforts are being made by the competitive swimmers to set new records. Therefore, in order to swim faster, there must be more thrust generated by the swimmer and the drag which resists the swimmer must be should be decreased. Experimental tests which are done on the swimmers help to determine the propulsive forces generated by the swimmer but these are very costly and also complicated. Hence researchers have applied numerical techniques of computational fluid dynamics as an alternative to the experimental methods to determine the force coefficients around the hand and arm which closely resembled those which were determined experimentally. These CFD techniques are being used all over the world on competitive swimmers to increase their thrust to the water to swim faster and are tested at various angles of attack of the hand. $A$ motion study for the same is made here of how these techniques were applied to enhance the speed of the swimmers.
\end{abstract}

Keywords: CFD technique, force coefficients, thrust

\section{Introduction}

Competitive swimming is one of the most challenging sports to perform scientific research. Not only the research of human movement is quite complex, because human beings are not so determinists as other biomechanical systems; but also, assessing human beings in aquatic environment becomes even more as this is not their natural environment and other physical principles have to be considered. Swimming performance depends on the interaction of propulsive and resistive forces.

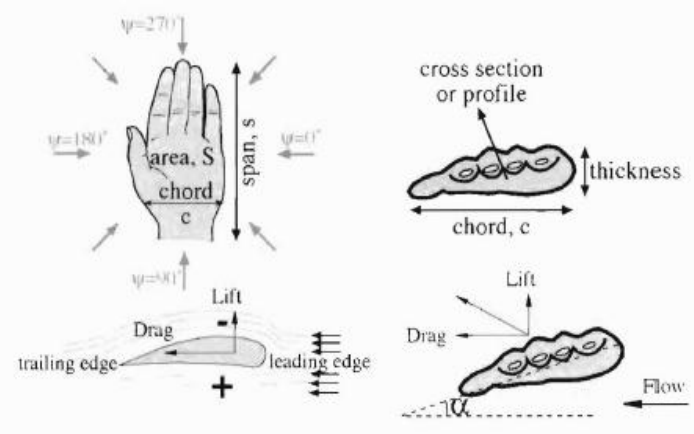

Fig.1a: Parameters describing the hand as hydrofoil

A swimmer can improve by reducing resistive forces, or drag, that acts on the swimming body at a given velocity or by increasing the propulsive forces. Front

*Corresponding author: Ameya K. Deshpande crawl is the fastest and most efficient swimming style. Arm propulsion is generated through alternating the sculling arm; while one of the arms is pushing/pulling the other is recovering. The arm stroke provides most of the forward momentum and supplies most of the propulsive force. At present the dominant view is that the hand acts as a hydrofoil, generating both lift and drag.

\section{Methods}

The major part of the thrust produced in the freestyle swimming (front crawl) depends on the angle of attack (the angle formed by the hand with the surface of water in the crawling direction) and the finger spacing on the hands. An optimum angle of attack and an optimum finger spacing increases the thrust produced by the swimmer and helps in competitive swimming. For analysing the effect of angle of attack on the thrust produced, Bixler and Riewald ${ }^{[4]}$ created a CFD model of hand and forearm with the wrist in neutral position. Using this, the lift and drag forces were determined by them and the hydrodynamic forces were evaluated and these force coefficients were validated by comparing them with the steady-state coefficients obtained experimentally.

The analysis done for the various angles of attack showed that the force coefficient was maximum for the angles of attack near $90^{\circ}$ and was minimum for the angles of attack between $120^{\circ}$ to $135^{\circ}$. From this the 
optimum angle of attack should be selected which should be selected based on the force coeffients where maximum drag can be reduced optimising the angle of attack.
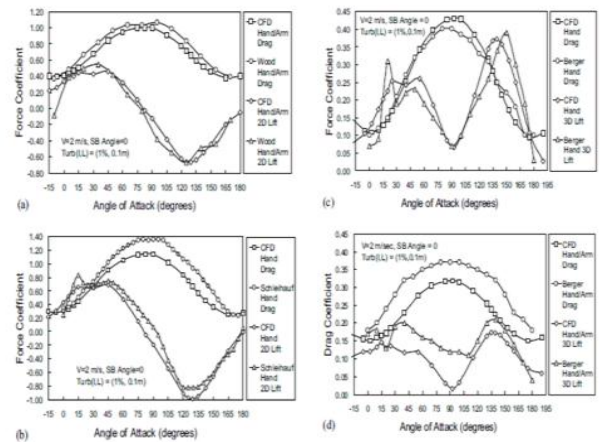

Fig. 2a: Variation of force coefficients with angles of attack

Also the in-between finger spacing on the hand has a effect on the thrust produced by the swimmer in the water. Therefore an analysis was done for the optimum finger spacing. It considered distinct styles of finger spacing and the results were evaluated for them accordingly. The optimum finger spacing is the one which produces maximum propulsion in the forward direction.

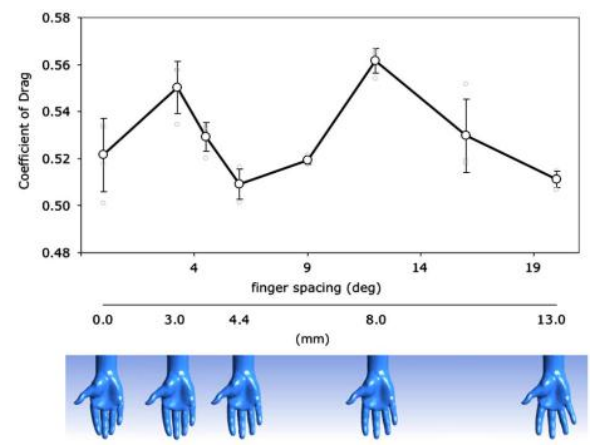

Fig. 2b: The upper graph represents the hand drag coefficients vs. different finger spacings, obtained by computation fluid dynamics simulations. Circles and vertical bars show average and SD values, respectively, calculated when pooling all the four investigated speeds.
The fig. 2(b) shows the variation of coefficient of drag with the finger spacing in angles as well as considering the length in between them. The optimal finger spacing is the one by which maximum drag can be produced by the swimmer for the forward crawl.

\section{Conclusion}

The experimental results validated the results obtained by CFD analysis of the hand and forearm of the swimmer which reduces the cost of experimentation and also the optimum finger spacing was studied to increase the thrust for swimming. Therefore the optimal finger spacing would be around $8.0 \mathrm{~mm}$ or $10^{\circ}$ to $12^{\circ}$ and the optimum angle of attack would depend on the force coefficient which is maximum around $90^{\circ}$ for drag coefficients and around $120^{\circ}$ to $135^{\circ}$ for lift coefficients. Based on the combination of both, optimal angle of attack and finger spacing, the propulsion speed can be increased.

\section{References}

Alberto E. Minetti , Georgios Machtsiras , Jonathan C. Masters . The optimum fingerspacing in human swimming. Journal of Biomechanics 42 (2009) 2188-2190.

Huub M Toussaint. Biomechanics of propulsion and drag in front crawl swimming,Institute of Fundamental and Clinical Human Movement Science, Vrije Universiteit, Amsterdam, The Netherlands(2002).

Tiago M. Barbosa ${ }^{1}$, Daniel A. Marinho,Mário J. Costaand António J. Silva Biomechanics of CompetitiveSwimming Strokes,Polytechnic Institute of Bragança/CIDESD, 2University of Beira Interior/CIDESD, Polytechnic Institute of Bragança/CIDESD, University of Trás-os-Montes and Alto Douro/CIDESD, Portugal.

Bixler, B., Riewald, S., 2002. Analysis of a swimmer's hand and arm in steady flow conditions using computational fluid dynamics. Journal of Biomechanics 35, 713-717 